\title{
Stock profesional, eficiencia educativa y segmentación: análisis de los procesos de formación profesional en Chile ${ }^{1}$
}

\author{
Professional Stock, educational efficiency and segmentation: \\ Process analysis of professional training in Chile
}

Recibido: 16 de enero de 2015- Revisado: 19 de mayo de 2015 - Aceptado: 20 de octubre de 2015

Carlos Rodríguez Garcés ${ }^{2}$

Víctor Castillo Riquelme ${ }^{3}$

\section{Resumen}

Este artículo describe y compara la posición relativa de las distintas carreras profesionales sobre un conjunto de índices educativos expresivos de los procesos formativos y atributos del contingente de titulados. Evento de relevancia en un escenario de educación superior como el chileno, caracterizado por altas tasas de cobertura y la presencia de una diversificada oferta de calidad segmentada. Estos índices informan de una alta tasa de titulados que hace prever problemas de empleabilidad y depreciación de las credenciales educativas; diferenciales niveles de retención y eficiencia educativa y un estructural proceso de elitización de la opción profesional. Comportamiento que es manifestación de un proceso de segmentación socioeducativa preexistente que se prolonga y profundiza en el espacio universitario.

\section{Palabras clave}

Capital humano, estratificación social, formación profesional, retención, deserción.

\begin{abstract}
This article describes and compares the relative position of the various professional careers in a set of education indexes expressive of traming processes and attributes of the group of graduates. It is a relevant event in a scenario of higher education as the chilean, characterized by high rates of coverage and the presence of a diversified range of segmented quality. These indexes report a high rate of graduates who do foresee problems of employability and depreciation of educational credentials; differential levels of retention and educational efficiency and structural process of elitization of the professional choice. This behavior is a manifestation of a pre-existing socio-educational segmentation process that extends and deepens the university space.
\end{abstract}

\section{Keywords}

Human capital, social stratification, professional training, retention, desertion.

\footnotetext{
${ }^{1}$ El presente artículo se enmarca dentro de la línea "Procesos de segmentación e inclusión socioeducativa" y del proyecto de investigación "El ranking de notas: Su efectividad como mecanismo de inclusión en la educación superior y su contribución a la capacidad predictiva del sistema de selección universitaria" siendo realizado por docentes investigadores de la Universidad del Bío-Bío de Chile. ${ }^{2}$ Doctor en Multimedia Educativa por la Universidad de Barcelona, Barcelona, España. Catedrático en la Universidad del Bío-Bío, Concepción, Chile y Director del Centro de Investigación y Desarrollo en Informática Educativa (CIDCIE).

Correo electrónico:

carlosro@ubiobio.cl

${ }^{3}$ Investigador asesor del Centro de Investigación y Desarrollo en Informática Educativa (CIDCIE) de la Universidad del Bío-Bío, Concepción, Chile. Correo electrónico: vcastillo@ubiobio.cl

Para citar este artículo use: Rodríguez, C., \& Castillo, V. (2015). Stock profesional, eficiencia educativa y segmentación: análisis de los procesos de formación profesional en Chile. Revista Civilizar Ciencias Sociales y Humanas, 15(29), 183-196.
} 


\section{Introducción}

El sistema actual de educación superior en Chile sienta sus bases en la reforma educativa de los años ochenta caracterizada por conceder una importancia indebida a los mecanismos de mercado en la provisión, regulación y calidad del servicio educativo. La gran demanda por estudios superiores, la falta de regulaciones en el mercado educacional, así como el fuerte interés del proveedor privado por participar en dicho mercado se traducen en una fuerte expansión de la oferta educativa, aumento de las tasas de matrícula y el número de egresados, dando lugar a una proliferación de programas e instituciones que ofrecen estos servicios educativos a un heterogéneo contingente de alumnos de capacidades y habilidades muy variadas en correspondencia con los segmentados niveles de exigencia y calidad de los mismos procesos de formación (Rodríguez \& Castillo, 2014).

Una diversificada y extendida presencia de instituciones y programas se instala a lo largo y ancho del territorio nacional, otorgando títulos y grados en las distintas áreas del conocimiento disciplinar. Y si bien el acceso se democratiza por cuanto siete de cada diez estudiantes de educación superior son primera generación, esta mayor cobertura se hace con base en la segmentación socioeducativa, la reducción de los niveles de selectividad y calidad de los procesos formativos, así como al robusto endeudamiento de los estudiantes o sus familias para financiar los altos costos que esta educación conlleva.

La masificación e incremento de cobertura de una educación superior cada vez menos selectiva (Ávalos, 2010) no solo ahonda los problemas de calidad y pertinencia de la labor formativa, sino que además trae aparejado un eventual sobrestock profesional. Anualmente, una cuota cada vez mayor de nuevos titulados busca ingresar al mercado laboral impactando en la estructura de salarios y condiciones de empleo. En efecto, aunque este es un tema con- troversial o de debate, el crecimiento inorgánico de la masa de titulados presionaría a la baja los salarios, reduciría las tasas de empleabilidad y precarizaría las condiciones de empleo producto de la depreciación de las credenciales educativas, acelerando mecanismos de subutilización de capital humano (Camarena \& Velarde, 2009; Meller \& Rappoport, 2003; Rama, 2009).

A ello se suma la irrupción de la empleabilidad extrínseca, entendida como la tendencia cada vez mayor de emplearse en actividades que poco tienen que ver con la formación profesional recibida. Fenómeno que si bien tiende a ser transversal, se manifiesta con mayor intensidad en determinadas áreas profesionales. Eventual sobrestock profesional que por la vía del aumento inorgánico de la matrícula afecta con más intensidad a ciertas ramas y especialidades de la formación profesional.

El crecimiento de la matrícula impacta y modifica las necesidades educativas y el perfil del estudiante que accede a la educación universitaria (Ministerio de Educación -Mineduc-, 2012). Como resultado del significativo engrosamiento de la matrícula femenina, sector poblacional que históricamente evidenciaba una menor participación en estas instancias educativas, se constata un proceso de feminización de la educación superior en su conjunto, aunque claramente acentuado en ciertas áreas del conocimiento y programas formativos.

Por otra parte, asociado a las condiciones de educabilidad y trayectorias escolares, se constata un acrecentamiento relevante en las tasas de deserción o abandono de los estudios. El acceso casi irrestricto a un sistema escasamente regulado deriva de modo inexorable en la problemática por retener al gran contingente de nuevos estudiantes en los programas e instituciones a los que ingresaron. El fenómeno de la deserción no se comporta de manera equitativa, pues si bien la ampliación de cobertura afecta de forma positiva la democratización del sistema educativo, son precisamente los estudiantes 
de primera generación en la educación superior quienes se enfrentan en mayor medida a las dificultades de adaptación al sistema.

Se ha evidenciado que los grupos con mayor desventaja socioeconómica tienen más alta probabilidad de no graduarse del programa que cursan o bien tardan más tiempo en completar sus estudios (Organización para la Cooperación y el Desarrollo Económicos -OCDE-, 2009; González \& Uribe, 2002). Esta situación acarrea una mayor reflexión acerca de la duración de los programas en Chile, que se sostiene en que los estándares internacionales apuntan a cursos significativamente más cortos y con menor carga de trabajo para los estudiantes y docentes (OCDE, 2009), y en la necesidad de establecer un conjunto de planes remediales de acción afirmativa, en especial los orientados a estudiantes de menor capital social y económico, a fin de mejorar los niveles de retención y eficiencia terminal de instituciones y programas.

Este nuevo escenario de masificación hace de la deserción un fenómeno socioeducativo más complejo, debiéndose diferenciar desde el punto de vista conceptual a aquellos que desertan voluntariamente de los que no (Himmel, 2002). La deserción forzada o involuntaria, por lo general fundada en el mal desempeño académico que conlleva la decisión institucional de solicitar el retiro del estudiante, cohabita con el abandono voluntario, es decir, aquel de carácter temporal realizado con el propósito de transferirse de institución o programa. Modalidad de abandono, esta última, que se puede observar con mayor intensidad que antaño en atención al amplio abanico de opciones que ofrece el mercado, el acceso menos restringido y los menores costos ligados a esta decisión.

En consecuencia, la deserción no siempre implica la marginación definitiva del sistema, sin embargo, las segundas oportunidades también parecen estar fuertemente segmentadas en función de las características socioeconómicas de los estudiantes. Dentro del grupo de discen- tes provenientes de colegios particulares, un $72,6 \%$ de quienes desertan vuelve a acceder al sistema, situación que contrasta de modo significativo con el caso de los egresados de colegios municipales, donde solo el $45,6 \%$ de los desertores se reintegra a la educación superior (Rolando, Salamanca, Lara, \& Blanco, 2012).

La modificación del perfil estudiantil actúa también sobre los niveles de aprobación y eficiencia terminal del sistema universitario. Los niveles de exigencia de los programas, las condiciones de educabilidad y la escasa flexibilidad de las mallas curriculares inciden en las trayectorias académicas del estudiante, reduciendo la eficiencia educativa del proceso formativo que se manifiesta en el retraso progresivo y ralentización del ritmo de los estudios que moderan a la baja la tasa de egresados. Fenómeno del rezago educativo, eficiencia terminal y éxito académico que evidencia un comportamiento diferenciado con base en la composición del estudiantado de la institución o programa de formación profesional.

Por tanto, la democratización del sistema universitario y aumento de sus tasas de cobertura privilegia el acceso, pero desatiende la preocupación por la permanencia y continuidad del estudiante, trasladando el fenómeno de la exclusión social al interior de sus aulas; situación que da lugar a la existencia de circuitos o trayectorias educativas diferenciadas según el origen social del reclutamiento (Tedesco, 1987), profundizando los procesos de segmentación socioeducativa existente en la base del sistema escolar chileno.

En efecto, la educación terciaria extiende las desigualdades escolares que de la pertenencia socioeconómica se derivan, a la vez que reproduce disparidades de género fortalecidas en la división sexual del trabajo. La dependencia del establecimiento, mediada por el efecto de composición socioeconómica, determina los niveles de aprovechamiento educativo expresados en las pruebas de selección universitaria, 
de forma que el acceso a carreras universitarias de alto prestigio es virtualmente inexistente en egresados del sistema público municipal, contingente relegado en mayor cuantía a programas de menor estatus y exigencia, así como a instituciones de menor prestigio académico. Como corolario natural de la segmentación socioeducativa se elitizan instituciones y programas con altos niveles de selectividad y nivel socioeconómico con fundamento en la rentabilidad esperada y prestigio social de la profesión o carrera.

En atención a los fenómenos esbozados resulta de interés conocer el comportamiento que tiene el sistema de formación profesional de carácter universitario en su dimensión socioeducativa. Se exploran así, junto con los niveles de stock profesional, las tasas de retención, eficiencia terminal, feminización y elitismo educativo, entendidos estos como efectos directos del proceso de masificación de la educación superior que modifica el perfil del alumnado y sus necesidades educativas, complejizando la labor formativa.

\section{Metodología}

Se realizó una investigación descriptiva de carácter cuantitativo, se calcularon medidas e índices estadísticos a partir de la base de datos: Titulados 2012, facilitada por el sitio web www. mifuturo.cl. Este portal depende del Mineduc y se especializa en proveer información para la toma de decisiones vocacionales en educación superior.

Este artículo describe, analiza y compara la posición relativa o ranking exhibido por distintas carreras del ámbito profesional sobre un conjunto de indicadores educativos construidos por los autores para representar cuantitativamente las manifestaciones de los fenómenos en estudio. La muestra del estudio agrupa a las 98 carreras profesionales impartidas por universidades e institutos profesionales en Chile. Estas carreras conducen a un título profesional, requiriendo para ello un mínimo de cuatro años de formación.
Los indicadores construidos buscan testear o dar cuenta de los perfiles educativos del mercado de la formación profesional existente en el país a través de la exploración del comportamiento de los siguientes índices:

a) Índice de titulados, expresa en términos absolutos la dotación de profesionales que se gradúan en un año determinado y que, por tanto, se constituyen en nueva fuerza de capital humano en el campo laboral que complementa el stock profesional existente en el mercado del trabajo.

b) Índice de retención de primer año, se obtiene mediante el cociente resultante entre el número de estudiantes que ingresan a primer año a una carrera determinada, y el número de esos mismos estudiantes que mantienen tal condición al año siguiente en la misma institución y programa. Esta tasa no informa de la proporción de la cohorte que en efecto culmina sus estudios, pero es importante en virtud de que la deserción se produce en mayor cantidad en los primeros semestres. Así mismo, tiene la limitante de que tampoco discrimina entre el abandono de carácter permanente o temporal, sea para cambiar de institución o de programa.

c) Índice de feminización, valor que toma el cociente entre la cantidad de mujeres tituladas y el total general de titulados. Es decir, estima la participación relativa de las mujeres en el sistema de educación superior para cada una de las carreras, dando cuenta de las diferencias de género en las elecciones profesionales y procesos formativos.

d) Índice de eficiencia terminal, diferencial relativo entre la duración real y la duración de referencia. El valor asociado a este índice expresa la propor- 
ción extra de la duración del programa que en promedio tarda un discente en completar sus estudios. Un valor superior a uno implica que en promedio los estudiantes demoran más del doble de lo teóricamente declarado en el programa. Por contraparte, valores cercanos a cero señalan un buen ajuste entre la cantidad de semestres referenciales y la cantidad de semestres efectivamente cursados. Como expresión relativa de éxito académico es atributo del programa, de la institución y de los mismos alumnos, por cuanto manifiesta el grado de flexibilidad curricular, el compromiso institucional con el proceso educativo y el perfil y condiciones de educabilidad del alumno.

e) Índice de elitización, cociente resultante entre el número de estudiantes matriculados en una carrera determinada provenientes de colegios de dependencia particular pagada, y el número total de estudiantes matriculados para ese mismo año en igual institución y programa. Asumiendo que del total de educandos que ingresan al sistema de educación superior un $10 \%$ proviene de instituciones privadas, un índice superior/inferior a 0,1 estaría señalando una sobrerrepresentación/ subrepresentación de este segmento, siendo un indicador de la influencia del capital cultural y el nivel socioeconómico en las elecciones profesionales, así como una clara expresión de los procesos de segmentación socioeducativa que se da en el escenario de la educación superior en Chile.

\section{Análisis}

La educación, en especial la de nivel terciario o superior, entendida como proceso, genera un conjunto de resultados. Outcomes educativos o perfiles de egreso que, expresados en un set de índices, evidencian el diferenciador y segmentado panorama de la formación profesional en su fase terminal existente en Chile. En efecto, se observa un volumen de titulados cada vez mayor en atención a la masificación del sistema educativo; diferenciados niveles de retención y eficiencia terminal de las carreras profesionales con base en los perfiles de ingreso que segmentan las trayectorias académicas del alumnado, distorsionados niveles de exigencia, así como una variada oferta educativa que viabiliza, dentro de ciertos márgenes, las posibilidades de las segundas oportunidades en la elección profesional. Y por último, la elitización profesional como resultado de un proceso de segmentación socioeducativa que se manifiesta transversalmente en todo el sistema y se prolonga de modo natural en la educación superior, reproduce en sí misma las desigualdades sociales preexistentes y que por la vía del mayor prestigio y rentabilidad de la elección profesional tiende a perpetuarse.

Tabla 1 Índice de titulados

\begin{tabular}{|c|c|}
\hline \multicolumn{2}{|c|}{ Mayor stock de egresados } \\
\hline Ped. en Educ. básica & 4.827 \\
\hline Ing. comercial & 4.105 \\
\hline Adm. empresas & 3.232 \\
\hline Psicología & 3.167 \\
\hline Auditoría & 2.867 \\
\hline
\end{tabular}

\begin{tabular}{|l|l|}
\hline \multicolumn{2}{|c|}{ Menor stock de egresados } \\
\hline Ing. en Adm. industrial & 21 \\
\hline Bibliotecología & 21 \\
\hline Ing. en industrias de la madera & 25 \\
\hline Ing. en automatización & 38 \\
\hline Tecn. en análisis químico & 47 \\
\hline
\end{tabular}

Nota: Adm,=Administración; Ing.=Ingeniería; Ped=Pedagogía; Educ. $=$ Educación Tecn. $=$ Tecnología

Fuente: Elaboración propia a partir de Ministerio de Educación de Chile, 2012. 
Como resultado natural del incremento sostenido de la matrícula, que en la actualidad alcanza una cobertura bruta del $54,6 \%$ para el rango etario de 18 años a 24 años, se constata un permanente aumento del volumen de titulados que el sistema de educación superior aporta anualmente al mercado del trabajo técnico y profesional. Solo en el campo de la formación profesional en el 2012 egresaron un total de 70.777 nuevos titulados. Si bien la masificación de la educación impacta todas las áreas del conocimiento, sus efectos se han hecho sentir con mayor fuerza en determinadas profesiones cuyo volumen de egresados sobrepasa la expectativa racional que de la demanda del trabajo se deriva.

La formación en Educación General Básica es en este contexto el claro dominador, siendo la profesión que de lejos más egresados aporta al sistema con cerca de cinco mil titulados al año. Cifra que no guarda proporción con la tasa de reemplazo ni nuevas vacantes que requeriría esta profesión en atención a la cobertura casi universal que tiene la Educación Básica, ni con el nuevo perfil demográfico caracterizado por una reducción en las tasas de fecundidad (Rodríguez \& Castillo, 2014).

Alta masa de nuevos profesionales que al incorporarse al mercado laboral modifica el perfil educacional de la fuerza de trabajo, depreciando determinadas credenciales educativas, en especial las de menor nivel o estatus, sin que ello involucre necesariamente acentuación del desempleo. La desocupación en la población con estudios superiores completos se mantiene estable siendo solo levemente mayor en los titulados nóveles. Matices del desempleo producto de un efecto de composición de la fuerza de trabajo. Es decir, la tendencia que presenta la población joven hacia una mayor desocupación, con independencia relativa de sus credenciales educativas.

Como contraparte, se constata que hay un conjunto de carreras con una exigua inyección de profesionales al mercado del trabajo. Esto es producto de la existencia de programas de denominación sui géneris, algunos de los cuales no trascienden a la unidad educativa que los creó y dicta en exclusividad. Reducida presencia no amparada necesariamente en las restricciones de un mercado laboral incierto o saturado, sino más bien en el escaso atractivo que estas provocan en el nuevo contingente que accede a la educación superior. En un mercado educacional apenas regulado, cuando los fondos siguen a los alumnos, es la demanda la que estimula la oferta de nuevos o más programas de un mismo perfil. Demanda que es influenciada por la publicidad y el marketing académico, con acceso desigual a la información para la toma de decisiones, así como por ser conservadora en las elecciones vocacionales.

Tabla 2

Índice de retención de primer año

\begin{tabular}{|lc|}
\hline \multicolumn{2}{|c|}{ Mejor puntuadas } \\
\hline Medicina & 0,93 \\
\hline Geología & 0,92 \\
\hline Química y Farmacia & 0,89 \\
\hline Ing. Civil & 0,88 \\
\hline Obstetricia & 0,87 \\
\hline
\end{tabular}

\begin{tabular}{|lc|}
\hline \multicolumn{2}{|c|}{ Peor puntuadas } \\
\hline Ing. en Industrias de la Madera & 0,56 \\
\hline Actuación y Teatro & 0,58 \\
\hline Ing. Industrial & 0,59 \\
\hline Ing. en Marketing & 0,61 \\
\hline Ing. en Electrónica & 0,61 \\
\hline
\end{tabular}

Nota: Ing.=Ingeniería

Fuente: Elaboración propia a partir de Ministerio de Educación de Chile, 2012.

El crecimiento sostenido de la masa de titulados es la consecuencia natural de la amplia cobertura alcanzada en educación superior, que se explica en especial por la proliferación de programas e instituciones de naturaleza privada, las cuales participan en el mercado educacional otorgando licenciaturas, títulos profesionales y 
certificaciones de nivel superior en las distintas áreas del conocimiento. Si bien la cobertura ha conseguido tasas históricas, el contingente de estudiantes que efectivamente logra egresar del sistema es moderado por los altos niveles de deserción que tiene la educación superior, fenómeno que hoy por hoy se constituye en uno de los principales problemas del sistema.

Aunque este abandono tiende a concentrarse en los primeros semestres, estabilizándose conforme se avanza en el programa académico, es una problemática de relevancia por su magnitud prevalente y fuerte segmentación socioeducativa, siendo expresión, esta última, de las debilidades que tiene el sistema para nivelar las competencias de entrada y procurar retener a alumnos de situación socioeconómica desmejorada.

En primer año, aparte de Actuación y Teatro, son las carreras de ingenierías las que registran menores tasas de retención estando por debajo del $70 \%$. Del grupo de carreras en análisis siete de cada diez de ellas ven desertar al menos al $20 \%$ de su alumnado en primer año, lo que da cuenta de lo extendida de la problemática. Por otra parte, carreras principalmente vinculadas al ámbito de la salud son las que evidencian los mayores índices de retención (Medicina, Química y Farmacia, Obstetricia y Odontología).

Un conjunto de elementos estaría asociado a los niveles de retención que muestra una carrera. Componentes que no se circunscriben única y exclusivamente a factores académicos o económicos, en términos de rendimiento deficiente o insuficientes recursos para solventar los costos que la educación implica; sino que se adentran también a factores motivacionales o de oportunidad.

La masificación de la educación superior y los cambios en los criterios de selectividad trajo consigo una transformación del perfil socioeconómico y académico del alumno y de sus necesidades educativas, situación que impactó fuertemente sobre los niveles de deserción, estimándose que en la actualidad alrededor del 50 $\%$ de los educandos no finaliza la carrera que inician (Mineduc, 2012).

Atendiendo a la magnitud y complejidad del fenómeno de la deserción es que, aquella de carácter involuntaria suscitada por motivos de rendimiento deficiente, falta de recursos y/o problemas de adaptación, está siendo abordada por una serie de medidas implementadas por las instituciones de educación superior a fin de disminuir las pérdidas de eficiencia del sistema educativo y ampliar las oportunidades de los estudiantes y sus familias, en particular en los quintiles más bajos. Es así como las mallas de las carreras han mutado hacia una mayor flexibilidad, restringiéndose los prerrequisitos, modificando las escalas evaluativas, reduciendo los grados de exigencia, semestralizando y modulando las asignaturas, así como un conjunto de otras acciones remediales con el propósito de aminorar los niveles de reprobación y abandono académico. Por otra parte, se ha instalado todo un sistema de créditos y becas de diversa índole y envergadura para cubrir los costos educativos.

Pero junto a estos factores de flexibilización académica y beneficios económicos para favorecer la retención estudiantil que se extiende a la generalidad del sistema, también actúan otras fuerzas de sentido inverso. La amplia y heterogénea oferta educativa y los bajos niveles de selectividad que evidencian un ámbito de formación disciplinar específico tendrían el efecto de incrementar la deserción académica de carácter transitoria, es decir, abandonan temporalmente un programa para incorporarse en corto plazo a otro. Deserción voluntaria que se activa con más vigor en programas e instituciones menos selectivas.

Por tanto, el abandono o deserción temprana de un estudiante de educación superior no es expresión lineal de la dificultad del programa cursado, sino que además es resultado de los 
costos de inversión inicial en que el estudiante haya incurrido para acceder a dicha institución o programa. Conforme el esfuerzo desplegado para acceder a un programa selectivo, que a su vez asegura altas tasas de retorno a la inversión realizada, torna más difícil la toma de decisión de abandonar el sistema, persistencia que se constata a pesar de las tasas de reprobación.

Tabla 3

Índice de feminización

\begin{tabular}{|lc|}
\hline \multicolumn{2}{|c}{ Mejor puntuadas } \\
\hline Ped. Educ. párvulos & 1 \\
\hline Diseño de vestuario & 0,96 \\
\hline Ped. en Educ. diferencial & 0,96 \\
\hline Psicopedagogía & 0,91 \\
\hline Nutrición & 0,91 \\
\hline
\end{tabular}

\begin{tabular}{|lc|}
\hline \multicolumn{2}{|c|}{ Peor puntuadas } \\
\hline Ing. mecánica automotriz & 0,01 \\
\hline Ing. en automatización & 0,03 \\
\hline Ing. en electricidad & 0,03 \\
\hline Ing. mecánica & 0,03 \\
\hline Ing. civil eléctrica & 0,04 \\
\hline
\end{tabular}

Nota: Educ.=Educación; Ing.=Ingeniería; Ped=Pedagogía.

Fuente: Elaboración propia a partir de Ministerio de Educación de Chile, 2012.

Tradicionalmente, la educación superior era un espacio de predominancia masculina, constituyéndose en un escenario de privilegios y reproductor de las desigualdades de género fortalecidas en la división sexual del trabajo, situación que se ha comenzado a revertir dando lugar a un acelerado proceso de feminización de la educación superior en los distintos ámbitos disciplinares, aunque en algunas profesiones esta participación femenina tiende a ser más relevante. Así por ejemplo, históricamente en Educación de Párvulos, Educación Diferencial y Enfermería han prevalecido altos índices de feminización, cuando no ser carreras en exclusiva de mujeres. Carreras que al año 2013 ocupaban posiciones levemente superiores a las de Trabajo Social (8) y Pedagogía en Educación Básica (10). Componentes de discriminación de género de matriz histórica, aspectos aptitudinales de carácter motivacional y características del mercado del trabajo explicarían este sesgo de género.

En principio, el acceso a la educación superior para las mujeres fue restringido y segregado a un conjunto limitado de profesiones $\mathrm{u}$ oficios. Circunstancia que, si bien se ha atenuado con el paso del tiempo, persisten resabios institucionales que no favorecen la incorporación femenina, tal es el caso de algunas especialidades del ámbito de las ingenierías, muchas de las cuales son de marcada o casi exclusiva presencia masculina.

En consecuencia, se reconoce la influencia cultural que reciben las mujeres por determinantes socializadores establecidos por la familia, los medios de comunicación y la sociedad en la elección de oficios y profesiones que se consideran aptos para su género. Factores que tienden a desalentar ciertas elecciones profesionales, en particular en aquellas de carácter gerencial y diferenciados niveles jerárquicos donde la competencia está dominada por varones, escenario en el cual a la mujer se le hace difícil competir con independencia de sus niveles de calificación, logro o mérito. Obstáculo conocido comúnmente en la literatura como "techo de cristal" (Organización Internacional del trabajo-OIT, 2004), el cual es reforzado por una desigual distribución de roles en el espacio doméstico, responsabilidad que asume mayoritariamente la mujer y que dificulta la conciliación con el mundo del trabajo.

Por otra parte, factores motivacionales de perfil aptitudinal de determinadas profesiones que tratan sobre el cuidado de personas influyen para que dichas carreras sean preferidas por mujeres, tal es el caso de aquellos oficios que tienen por objeto la enseñanza, el cuidado de niños o enfermos, entre otros. Perspectivas teóricas que señalan una predisposición en las mujeres 
hacia carreras sensibilizadoras relacionadas con el cuidado de personas y los servicios sociales. En efecto, la presencia femenina es el claro dominador en el conjunto de profesiones que versan sobre educación infanto-adolescente (Educación de Párvulos, Pedagogía Diferencial y Pedagogía General Básica), el cuidado de la salud de las personas (Nutrición, Obstetricia y Enfermería) y la prestación de servicios sociales (Trabajo Social y Psicología).

Por último, características del mercado del trabajo presionan a las mujeres a tener una mayor propensión hacia carreras más compatibles con estereotipos sociales de género de matriz reproductiva, para hacer más conciliable la ejecución del rol dual del cuidado del hogar y los hijos con la participación en el mercado laboral. Esto último explicaría a juicio de Meller (2010), que las mujeres se inclinen por profesiones de mayor flexibilidad en jornada de trabajo y horario, y que tiendan a penalizar en menor medida la discontinuidad de las trayectorias laborales. Atributos asociados a disciplinas de vocación social y de salud que, por lo demás, correlacionan con posiciones de menor estatus, valoración social y remuneración. En cambio las carreras de ciencia y tecnología, que requieren de mayores habilidades matemáticas y que se inscriben en estamentos de más alta jerarquía y poder en la toma de decisiones, tienen mayor tendencia hacia el componente masculino.

La matriz de compatibilidad de rol de género incidiría incluso en la elección del tipo y naturaleza del programa de especialización o de educación continua. Se observa una importante prevalencia femenina en estudios de diplomados y postítulos (64\%), que contrasta con lo observado en programas de doctorado y magíster (46\%). Elementos asociados a la flexibilidad y duración de dichos programas estarían en la base explicativa de estas diferenciales opciones entre hombres y mujeres.

Esta concentración de las mujeres en determinadas profesiones, si bien se mantiene como tendencia, se atenúa y suaviza con el tiempo, de forma tal que, salvo contadas excepciones, ya no se puede hablar de carreras exclusivamente masculinas o femeninas. Gran parte del aumento de la matrícula en la educación superior se explica por la participación de mujeres en este nivel de enseñanza irrumpiendo en todos y cada uno de los ámbitos disciplinares de formación, incluso en carreras históricamente hostiles como las ingenierías. Así por ejemplo, entre los años 2000 y 2006 el número de mujeres ingenieras tituladas se duplicó. De igual manera la participación masculina en carreras como Enfermería y Trabajo Social ha ido en ascenso en los últimos decenios.

Tabla 4

Índice de eficiencia terminal

\begin{tabular}{|lc|}
\hline \multicolumn{2}{|c|}{ Mejor puntuadas } \\
\hline Medicina & 0,08 \\
\hline Ing. en Finanzas & 0,09 \\
\hline Ing. en recursos humanos & 0,11 \\
\hline Ped. en Educ. diferencial & 0,15 \\
\hline Fonoaudiología & 0,16 \\
\hline
\end{tabular}

\begin{tabular}{|lc|}
\hline \multicolumn{2}{|c|}{ Peor puntuadas } \\
\hline Ing. en automatización & 1,31 \\
\hline Tec. en análisis químico & 0,96 \\
\hline Ing. en industrias de la madera & 0,93 \\
\hline Ing. forestal & 0,86 \\
\hline Ing. en química & 0,82 \\
\hline
\end{tabular}

Nota: Educ.=Educación; Ing.=Ingeniería; Ped=Pedagogía; Tec. $=$ Tecnología

Fuente: Elaboración propia a partir de Ministerio de Educación de Chile, 2012.

El índice de eficiencia terminal del programa de estudio, esto es, el diferencial relativo entre el promedio de semestres en que está distribuido el plan curricular de la carrera incluido el proceso de titulación (duración de referencia) y el promedio de semestres que efectivamente demoran los estudiantes desde que ingresan a la carrera hasta que se titulan (duración real), da cuenta de un heterogéneo comportamiento 
según el tipo de carrera que se trate. Aunque este índice es liderado por medicina, carrera altamente selectiva y de reconocida exigencia académica, son las profesiones de formación pedagógica las que predominan dentro de las carreras con menor diferencial temporal entre duración de referencia y duración real de sus planes de estudio. En efecto, entre las diez carreras de mayor eficiencia terminal encontramos cuatro profesiones del ámbito de la educación. En ellas su masa de titulados no supera la barrera temporal del $25 \%$ adicional a la duración de referencia de sus respectivos programas.

Como contraparte, carreras de ingenierías y del ámbito de las ciencias duras registran los peores índices a este respecto, donde sus estudiantes pueden demorar cerca del doble del tiempo esperado en titularse.

Cabe señalar que este índice recoge la influencia tanto de las particularidades de la estructura del plan de estudio como del perfil académico del estudiante. Así por ejemplo, medicina es una carrera fuertemente selectiva y de alta exigencia que al reclutar a alumnos de alto y homogéneo perfil educativo viabiliza trayectorias académicas más eficientes sin pérdida de calidad, con los consiguientes mayores niveles de promoción y retención de su estudiantado.

Por su parte, la posición que tienen las carreras pedagógicas, que se mantienen en el decil de mejor rendimiento en este índice, se explicaría más por las características del programa que por los atributos de su contingente estudiantil. Estas profesiones evidencian una proliferación de programas, muchos de ellos escasamente selectivos que admiten alumnos con bajo capital cultural y reducidos puntajes en las pruebas de selección universitaria (Ávalos, 2010), provenientes de sectores socioeconómicos medio y medio bajo, de bajo rendimiento escolar en la enseñanza media $y$, en una proporción significativa, son primera generación en la educación superior (Ávalos, 2010; Manzi, 2010).
Elementos que posibilitan señalar que la eficiencia educativa de dichos programas se asocia a los moderados, cuando no bajos, niveles de exigencia y calidad de sus procesos formativos con diferenciales sistemas evaluativos que tenderían a privilegiar la promoción con el fin de aumentar sus índices de eficiencia interna. En estas carreras, al contrario de medicina, las deserciones son altas y se producen principalmente durante el primer año. Sus niveles de reprobación, por su parte, están muy acotados y modelan a la baja como resultado de los planes de mejoramiento y convenios de desempeño que las instituciones se encuentran ejecutando. Y si bien mejoran en sus niveles de eficiencia interna, aún no son capaces de transferir dicha eficiencia a la calidad de sus procesos formativos.

Los deficientes resultados de la Prueba Inicia ${ }^{1}$ en sus distintos componentes estructurantes son expresión palpable de las dificultades que evidencia la instalación de competencias pedagógicas y disciplinares de calidad en el proceso de formación inicial docente, situación que opera con independencia relativa del rendimiento académico previamente evidenciado por el programa educativo en dicha casa de estudios.

Para el año 2011 en la prueba de conocimientos disciplinares un $69,2 \%$ de los profesores nóveles que rindieron la Prueba Inicia calificó insuficiente, es decir, no superó la barrera del estándar exigido en el dominio de los contenidos de la materia que han de enseñar (Rodríguez \& Castillo, 2014). Bajo nivel de logro a pesar de que el $85 \%$ y el $48 \%$ de los profesores recién egresados que rindieron la prueba registraban promedios ponderados anuales sobre 5,0 y 5,5 respectivamente en escala de 1 a 7. Esto es, alumnos titulados certificados de buen rendimiento por sus casas de estudio, pero que en un número significativo de ellos no revalidó dicha situación en la evaluación externa. Hecho que dista del constatado en los sistemas evaluativos ex post a los que son sometidos los facultativos médicos ${ }^{2}$. 
Tabla 5 Índice de elitización

\begin{tabular}{|c|c|}
\hline \multicolumn{2}{|c|}{ Mejor puntuadas } \\
\hline Diseño & 0,60 \\
\hline Medicina & 0,50 \\
\hline Ing. civil & 0,45 \\
\hline Ing. comercial & 0,41 \\
\hline Periodismo & 0,40 \\
\hline
\end{tabular}

\begin{tabular}{|lc|}
\hline \multicolumn{2}{|c|}{ Peor puntuadas } \\
\hline Ing. en finanzas & 0,01 \\
\hline Ing. agroindustrial & 0,01 \\
\hline Tec. en análisis químico & 0,01 \\
\hline Ing. en industrias de la madera & 0,02 \\
\hline Pedagogía en Educ. diferencial & 0,02 \\
\hline
\end{tabular}

Nota: Educ. $=$ Educación; Ing. =Ingeniería; Tec. $=$ Tecnología

Fuente: Elaboración propia a partir de Ministerio de Educación de Chile, 2012.

El acceso a la educación superior contribuye a la movilidad social por medio del mejoramiento de los niveles de ingreso $\mathrm{y}$, en la medida que estos niveles de renta están diferenciados por el tipo de profesión, las elecciones vocacionales están hondamente influenciadas por la rentabilidad futura de la carrera estudiada. Por otra parte, cuando determinados niveles de educación y tipos de formación profesional están restringidos a un segmento reducido y privilegiado de la población, el acceso a la educación superior se convierte en un mecanismo de reproducción de disparidades preexistentes. Es así como carreras de alto estatus y reconocimiento social, que a su vez evidencian mejores grados de empleabilidad y renta, tienden a ser preferidas por el segmento de mayor nivel socioeconómico de la población.

Inversa situación acontece en profesiones de menor estatus y renta. Así por ejemplo, alrededor del $10 \%$ de la población que ingresa a la educación superior proviene de colegios particulares pagados, pero en carreras como medicina esta proporción alcanza al 50,2 \%, ocupando el segundo puesto entre 98 carreras; en pedagogía en educación diferencial en cambio, esta cifra solo alcanza el $2 \%$. Es decir, en el mercado educacional la opción profesional estaría condicionada por componentes de renta y estatus, y no solo por exclusivas elecciones vocacionales, dando cuenta de la elasticidad de la demanda por cuestiones de rentabilidad futura y prestigio.

Esta sobrerrepresentación de titulados de alto capital sociocultural y económico en determinadas disciplinas de élite, y como corolario una subrepresentación en otras de menor prestigio, no solo recoge la influencia del campo disciplinar en su relación con el mercado laboral, sino que además del tipo de universidad de que se trate, razón por la cual la segregación socioeducativa del alumnado tiende a aumentar dentro de una misma carrera cuando estas pertenecen a diferentes instituciones educativas.

El aumento de cobertura democratizó el acceso a la educación superior haciendo de la condición social de universitario un lugar común con independencia relativa del nivel socioeconómico, no obstante constatarse profundos sesgos en los procesos de selección, retención y promoción del estudiantado. Pervive una estructura de oportunidades segmentada que reproduce las desigualdades instaladas en la base del sistema educativo y las prolonga al interior de ciertas instituciones y programas formativos de educación superior. Existe, en consecuencia, una formación profesional elitizada socialmente con restricciones de acceso no solo fundamentadas en puntuaciones obtenidas en los exámenes de selección universitaria, niveles de logro por lo demás condicionados por el capital cultural y económico familiar del alumnado; sino que también restringida por los altos costos de matrícula y modalidad de financiamiento.

Superadas las barreras de acceso para entrar a universidades selectivas, el sistema se encarga de ahondar aún más las brechas 
interuniversitarias por medio de un modelo de financiamiento indirecto o subsidio a la demanda que adiciona recursos por tramos de puntaje en el test de admisión alcanzado por el estudiante, de forma que se focaliza el gasto en las universidades que concentren a la élite escolar, aun cuando esta correlaciona con el capital económico y cultural.

Elitización de instituciones y programas por la vía de la configuración de su alumnado que pone en cuestión el rol movilizador social y meritocrático de la educación. En efecto, determinadas instituciones de educación superior y carreras profesionales se caracterizan por captar alumnos pertenecientes a los mayores segmentos de ingresos dotados de un capital cultural superior, mejor rendimiento escolar y mayor puntaje en las pruebas de admisión, mientras otras reclutan al contingente estudiantil de menores ingresos, muchos de ellos primera generación en la educación superior, con experiencias educativas de deficiente calidad $\mathrm{y}$, por lo mismo, con mayores dificultades para articular adecuados procesos de transición al ámbito universitario.

Esta estratificación socioeducativa de la educación superior, acentuada en los extremos de la distribución (heterogeneidad intergrupos) y caracterizada por una marcada homogeneidad intragrupo, hace que las instituciones educativas pierdan su mixtura social y la capacidad de ser el espacio de encuentro sociocultural. Es precisamente la segmentación socioeconómica instalada transversalmente en el modelo educativo chileno, y agudizada en la educación superior, la principal fuente de las críticas que levantan las movilizaciones estudiantiles y los diversos círculos académicos e intelectuales en Chile en los últimos quince años.

En síntesis, con base en los índices educativos podemos constatar que el espacio de la formación profesional universitaria en Chile no solo es amplio en cuanto al número de programas, matrícula y stock de nuevos profesionales que se insertan al mercado del trabajo, sino que además los procesos formativos difieren de forma significativa en cuanto a niveles de eficiencia terminal, retención y elitización educativa, dando cuenta de la gran heterogeneidad del sistema, sea desde el punto de vista de la oferta como de la demanda.

\section{Conclusiones}

Los procesos de reforma educativa que iniciaron en los años ochenta han favorecido la proliferación de instituciones y programas de educación superior $\mathrm{y}$, con ello, el aumento sustancial del stock de nuevos profesionales que buscan insertarse al mercado del trabajo. Racionalidad del proceso formativo con fundamento en la demanda y no necesariamente condicionada por los requerimientos de la actividad productiva, situación que puede implicar en el mediano plazo el sobrestock profesional, la depreciación de las credenciales educativas y la reducción de los niveles de empleabilidad e ingresos. Problemática que manifestará comportamientos diferenciados con base en el tipo de opción profesional y centro de estudio elegido. Aunque este es todavía un tema controversial, en lo inmediato se constatan mercados de trabajo con preocupantes niveles de saturación, en especial en un conjunto de profesiones vinculadas al ámbito de la educación y del derecho.

Las tasas históricas de cobertura han modificado el perfil de ingreso del estudiantado y diversificado la oferta educativa, incrementando con ello los niveles de deserción y alterando los niveles de eficiencia terminal. El abandono académico y la eficiencia educativa terminal si bien moderan la tasa de titulados, se constituyen en una de las principales problemáticas del sistema, siendo expresión de la falta de rigor en los procesos de selección, de las debilidades de la formación recibida y de la escasa capacidad del sistema para allanar los déficits en las competencias de entrada del estudiante. 
Se suma a ello que la proliferación y diversificación de la oferta otorga mayor viabilidad al abandono transitorio, haciendo de los cambios de programa o carrera opciones menos costosas, aunque estas segundas oportunidades están fuertemente segmentadas según el perfil socioeconómico y educativo del alumno. Falta de eficiencia terminal que no solo encarece los costos privados y públicos de la educación, sino que además frustra las expectativas por la pérdida de opción o chance de acceder a adecuadas credenciales educativas que le posibiliten al estudiante insertarse en el mercado del trabajo y generar rentabilidad de la inversión educativa realizada.

Situación que se constituye en un daño emergente, presente y futuro, en especial para los primeros quintiles, máxime cuando la inversión económica ha sido mediante créditos contraídos con entidades bancarias que inobjetablemente deberá cancelar el discente. Así entendido, este evento enfrenta al desertor estudiantil al peor de los mundos: contrae una deuda de egresado universitario, con las credenciales educativas de un estudiante de enseñanza media.

El espacio de la formación profesional se ha democratizado con la fuerte irrupción de la mujer. La participación femenina está segmentada en distintos tipos de carreras, prevaleciendo con mayor intensidad en aquellas vinculadas a la educación y la salud. Áreas que aportan anualmente al mercado laboral un contingente importante de nuevas egresadas. No obstante ello, en otras ramas que históricamente le han sido hostiles, tales como las ingenierías, las mujeres poco a poco ganan presencia, por lo que ya no se puede hablar de carreras en exclusiva masculinas. Proceso de feminización de la educación superior que se desprende como corolario natural de la fuerte inserción de la mujer al mercado del trabajo.

Mujeres cada vez más empoderadas retardan la nupcialidad, planifican y postergan la llegada de los hijos y formulan proyectos de vida no estrictamente asociados a la constitución de pareja; independencia que se sustenta en los ingresos del trabajo remunerado, los cuales se sujetan, en estabilidad y cuantía, al tipo y calidad de la formación recibida. Por ello, una cuota cada vez más alta de mujeres demanda y accede a una mayor y mejor educación.

La formación profesional se elitiza y segmenta de acuerdo con el perfil socioeconómico de la demanda. La elección profesional no es una mera expresión de una opción de tipo vocacional. Esta se entiende también como una decisión de inversión que busca rentabilidad, razón por la que, las profesiones que registran ingresos superiores, estatus y reconocimiento social, tienden a ser preferidas por el segmento de mayor nivel socioeconómico. Como resultado de esto, el mercado educacional se elitiza en términos de institución y carrera, restringiendo el acceso de estudiantes con diferente perfil sociocultural conforme aumenta el prestigio y estatus de la institución y programa.

Sesgo que reduce la capacidad de movilidad vertical que se le atribuye a la educación superior en sociedades meritocráticas $\mathrm{y}$, en su defecto, tiende a reproducir las desigualdades no remediadas por el sistema escolar chileno, ahondando los procesos de segmentación. La verificación de este hecho, por una parte, hace de la acción formadora de carácter superior una tarea compleja, donde la instalación de competencias profesionales para el mercado del trabajo enfrenta las restricciones de las condiciones de educabilidad de la masa estudiantil que se atiende.

Por otra parte, desaprovecha los talentos académicos existentes en otros segmentos sociales que al estar expuestos a ambientes privativos socioculturalmente carecen de los estímulos que permitan que las potencialidades se manifiesten en toda su extensión, evidenciándose así un marcado sesgo de selección que restringe la opción profesional de mayor estatus a la población estudiantil proveniente del sistema 
de enseñanza particular pagado, convirtiendo la elección vocacional en una tradición familiar que se transmite y hereda de padres a hijos.

En virtud de lo expuesto se hace necesaria una mayor regulación del mercado educativo de nivel superior y una reorientación de las prioridades por parte de la política educativa, por cuanto estas tienen el potencial de hacer más plausible, eficiente y racional la formación profesional en términos de acceso, calidad $\mathrm{y}$ pertinencia que asegure un adecuado y eficiente proceso de inserción laboral del futuro profesional. Regulación que, por lo demás, resguarda el interés público por sobre el privado y limita la posibilidad de abuso en que incurren ciertos emprendimientos en el mercado educativo que usufructúan de las expectativas de la demanda de quienes buscan mejorar sus competencias para el mercado del trabajo.

\section{Notas}

${ }^{1}$ En promedio un $60 \%$ de los profesores nóveles egresados de universidades chilenas reprueba en la Prueba Inicia.

${ }^{2}$ Tan solo un 2,9 \% de los médicos nóveles egresados de universidades chilenas reprueba en el Examen Único Nacional de Conocimientos de Medicina.

\section{Referencias}

Ávalos, B. (2010). Formación inicial docente en Chile: calidad y políticas. En C. Bellei, D. Contreras \& J. Valenzuela (Eds.), Ecos de la revolución pingüina: Avances, debates $y$ silencios en la reforma educacional (pp. 257-284). Santiago de Chile: Pehuén Editores.

Camarena, B. \& Velarde, D. (2009). Educación superior y mercado laboral: vinculación y pertinencia social ¿Por qué? y ¿Para qué?. Estudios sociales, 1, 106-125.
González, L. \& D. Uribe (2002). Estimaciones sobre la repitencia y deserción en la educación superior chilena. Consideraciones sobre sus implicancias. Revista de la $\mathrm{Ca}$ lidad de la Educación. 17, 75-90.

Himmel, E. (2002). Modelos de Análisis de la Deserción Estudiantil en la Educación Superior. Calidad en la Educación, 17, 91-108.

Manzi, J. (2010). Programa INICIA: fundamentos y primeros avances. En C. Bellei, D. Contreras \& J. Valenzuela (Eds.), Ecos de la revolución pingüina. Avances, debates y silencios en la reforma educacional (pp. 285-310). Santiago de Chile: Pehuén Editores. Recuperado de http://www.ub.edu/obipd/PDF\%20 docs/Formaci\%C3\%B3\%20Inicial/ Educaci\%C3\%B3\%20Universitaria/Documents/Ecos\%20de\%201a\%20revolucion.pdf

Meller, P. (2010). Carreras universitarias: rentabilidad, selectividad y discriminación. Santiago de Chile: Uqbar Editores.

Meller, P., \& Rappoport, D. (2003). Comparaciones internacionales de la dotación de profesionales y la posición relativa chilena. Santiago de Chile: Centro de Economía Aplicada, Universidad de Chile.

Ministerio de Educación-[MINEDUC]. (2012). Deserción en la educación superior en Chile (MINEDU, Serie Evidencias Ministerio de Educación No. 9 año 1). Recuperado de http://centroestudios.mineduc. cl/tp_enlaces/portales/tp5996f8b7cm96/ uploadImg/File/A1N9_Desercion.pdf

Organización Internacional del Trabajo -[OIT] (2004). Romper el techo de cristal: Las mujeres en puestos de dirección. Ginebra: Oficina Internacional del Trabajo. 
Organización para la Cooperación y el Desarrollo Económicos -[OCDE]. (2009). La educación superior en Chile (OCDE, Revisión de políticas nacionales de educación). Santiago de Chile: OECD; Word Bank. Recuperado de http://www.oecdilibrary.org/education/revision-de-politicas-nacionales-de-educacion_1990021x

Rama, C. (2009). La tendencia a la masificación de la cobertura de la educación superior en América Latina. Revista iberoamericana de educación, 50, 173-195.

Rodríguez, C. \& Castillo, V. (2014). Calidad en la formación inicial docente: los déficits de las competencias pedagógicas y disciplinares en Chile. Revista electrónica Actualidades Investigativas en Educación, 14(2), 1-25.

Rolando, R., Salamanca, J., Lara, A. \& Blanco, C. (2012). Deserción y reingreso a Educación Superior en Chile. Análisis de la Cohorte 2008. Santiago de Chile: Servicio de Información de Educación Superior -[SIES].

Tedesco, J. C. (1987). Modelo pedagógico y fracaso escolar. Revista de la CEPAL, 21, 131-145. 
